WUE-ITP-96-005

hep-ph/9604439

\title{
Chargino-Sneutrino Production in Electron-Photon Collisions
}

\author{
S. Hesselbach* and H. Fraas \\ Institut für Theoretische Physik, Universität Würzburg \\ D-97074 Würzburg, Germany
}

(September 1996)

\begin{abstract}
We study the production of charginos and sneutrinos in electron-photon collisions $\left(e^{-} \gamma \rightarrow \tilde{\chi}_{j}^{-} \tilde{\nu}_{e}\right)$ within the minimal supersymmetric standard model (MSSM). The high energy photons can be generated by Compton backscattering of intense laser pulses off one of the beams of the Next Linear Collider (NLC). This process could offer a significant opportunity to identify sneutrinos, which exclusively decay invisibly into a neutrino and the LSP, since the cross sections are two orders of magnitude higher than for the radiative production of invisible sneutrinos $\left(e^{+} e^{-} \rightarrow \tilde{\nu} \overline{\tilde{\nu}} \gamma\right)$. For three scenarios of gaugino-higgsino mixing the cross sections and polarization asymmetries are computed and the resulting signatures are compared with the SM background.
\end{abstract}

PACS numbers: 14.80.Ly, 12.60.Jv, 13.10.+q, 13.88.+e

*email: hesselb@physik.uni-wuerzburg.de 


\section{INTRODUCTION}

As a characteristic feature of supersymmetry (SUSY) with R-parity conservation all superparticles decay into the stable lightest supersymmetric particle (LSP) which is usually assumed to be the lightest neutralino $\tilde{\chi}_{1}^{0}$. Since the LSP is weakly interacting, it escapes detection and carries missing energy $(\not E)$ as the most distinctive signature of SUSY events. It may, however, happen that the sneutrino is lighter than both the second lightest neutralino and the lightest chargino. In this case the only kinematically allowed two-body decay of the sneutrino is the invisible channel $\tilde{\nu}_{e} \rightarrow \nu_{e} \tilde{\chi}_{1}^{0}$. Light sneutrinos are allowed in supergravity models with common scalar and gaugino masses at the unification scale [1] and have been until now not experimentally excluded [2,3]. Then the only way to identify sneutrino production in $e^{+} e^{-}$-annihilation is the radiative process $e^{+} e^{-} \rightarrow \tilde{\nu} \tilde{\nu} \gamma$ [4]. In the energy region between 100 and $500 \mathrm{GeV}$, however, the cross sections for this process are rather small, of the order of $10^{-2} \mathrm{pb}$, so that due to the large irreducible background from $e^{+} e^{-} \rightarrow \nu \bar{\nu} \gamma$ it would be very difficult to identify an invisible sneutrino. Also the use of polarized beams cannot substantially improve the situation [4]. Recently Datta, Guchait and Drees [1] discussed in detail the impact of scenarios with invisible sneutrinos on SUSY searches at LEP 200, leading to dramatic effects on the signal for pair production of light charginos.

We study in this paper an alternative option: the associated production of light sneutrinos and charginos in electron-photon collisions $e^{-} \gamma \rightarrow \tilde{\nu}_{e} \tilde{\chi}_{j}^{-}$within the scope of the minimal supersymmetric standard model (MSSM) (see, e.g., [5]). Since radiative sneutrino pair production in $e^{+} e^{-}$-annihilation is a higher order process, the $e^{-} \gamma$-option should lead to drastically increased cross sections. Beyond that one expects for a light and invisible sneutrino a clear signature since in this case the light chargino decays almost completely leptonically into a single lepton and a neutrino. We give in the following the cross sections for $e^{-} \gamma \rightarrow \tilde{\nu}_{e} \tilde{\chi}_{j}^{-}$for photons produced by backscattering of laser pulses on a high energy electron beam [6, [] and confront scenarios with light invisible sneutrinos and heavy sneutrinos decaying visibly. Since experiments with suitable polarized beams may be helpful to discriminate between the supersymmetric process and the standard model (SM) background [8], we discuss the polarization asymmetries of the convoluted cross sections for different polarizations of the electron beam and the laser beam. For unpolarized and monochromatic photons this process has been investigated by Grifols and Pascual [9]. The authors confined themselves, however, to pure wino-like charginos with masses now experimentally excluded.

This paper is organized as follows: In sec. II we give the analytic formulae for the cross sections and polarization asymmetries in the electron-photon center of mass system (CMS) as well as in the laboratory system. After choosing three characteristic gaugino-higgsino scenarios we present in sec. III the numerical results for monochromatic photons and for the $e-\gamma$ mode of a linear collider. Finally we discuss the supersymmetric signatures in comparison with the SM background. 


\section{CROSS SECTIONS AND POLARIZATION ASYMMETRIES}

The Feynman graphs contributing to the process $e^{-}+\gamma \longrightarrow \tilde{\chi}_{j}^{-}+\tilde{\nu}_{e}(j=1,2)$ are shown in fig. 1. Denoting the four-momenta of $e^{-}, \gamma, \tilde{\chi}_{j}^{-}$and $\tilde{\nu}_{e}$ with $p, k, p^{\prime}$ and $k^{\prime}$, we introduce the Mandelstam variables $s=(p+k)^{2}, t=\left(k-p^{\prime}\right)^{2}$ and $u=\left(p-p^{\prime}\right)^{2}$. $\epsilon^{\mu}$ is the polarization vector of the photon, $S^{\mu}\left(S^{\prime \mu}\right)$ the spin vector of the electron (chargino).

The relevant couplings of the supersymmetric particles can be deduced from the following interaction Lagrangians of the MSSM (as for notations and conventions, we closely follow [5]):

$$
\begin{gathered}
\mathcal{L}_{e \tilde{\nu} e \tilde{\chi}}=-g \sum_{j=1}^{2}\left(V_{j 1}^{*} \overline{\tilde{\chi}}_{j}^{c} P_{L} e \tilde{\nu}_{e}^{*}+V_{j 1} \bar{e} P_{R} \tilde{\chi}_{j}^{c} \tilde{\nu}_{e}\right), \\
\mathcal{L}_{\tilde{\chi} \tilde{\chi} \gamma}=-e A_{\mu} \sum_{j=1}^{2} \bar{\chi}_{j} \gamma^{\mu} \tilde{\chi}_{j} .
\end{gathered}
$$

For completeness, we add the Lagrangian for the ee $\gamma$-coupling

$$
\mathcal{L}_{e e \gamma}=e A_{\mu} \bar{e} \gamma^{\mu} e .
$$

In equs. (1) - (3) $\tilde{\chi}_{j}(j=1,2)$ and $e$ are the four-component spinors of the chargino and the electron, while $\tilde{\nu}_{e}$ is the field of the electron-sneutrino. $\tilde{\chi}_{j}^{c}$ are charge conjugated spinor fields. Furthermore, $P_{R, L}=\left(1 \pm \gamma_{5}\right) / 2$ denote the right- and left-handed projection operators, $g=e / \sin \theta_{W}(e>0)$, and $V_{i j}$ is the $2 \times 2$ unitary matrix appearing in the diagonalization of the wino-charged higgsino mass matrix (see [10] for more details).

The differential cross section for circularly polarized photons with helicity $\lambda_{\gamma}$ and electrons with helicity $\lambda_{e}= \pm 1$ is given by

$$
\frac{d \sigma}{d \Omega}=\frac{d \sigma^{0}}{d \Omega}\left(1-\lambda_{e}\right)\left(1+\lambda_{\gamma} a_{c}\right)
$$

In the electron-photon CMS the cross section for unpolarized beams is

$$
\frac{d \sigma^{0}}{d \Omega}=\frac{\left|V_{j 1}\right|^{2} \alpha^{2}}{8 \sin ^{2} \theta_{W}} \frac{w}{s^{2}}\left\{\frac{2 s+4 \Delta_{j}\left(1+\frac{\Delta_{j}}{s}\right)}{s+\Delta_{j}-w \cos \theta}-\frac{8 m_{\tilde{\chi}_{j}^{ \pm}}^{2} \Delta_{j}}{\left(s+\Delta_{j}-w \cos \theta\right)^{2}}-\frac{3 s+3 \Delta_{j}+w \cos \theta}{2 s}\right\},
$$

and the circular polarization asymmetry $a_{c}$ is given by

$$
a_{c} \frac{d \sigma^{0}}{d \Omega}=\frac{\left|V_{j 1}\right|^{2} \alpha^{2}}{8 \sin ^{2} \theta_{W}} \frac{w}{s^{2}}\left\{\frac{2 s+4 \Delta_{j}+4 m_{\tilde{\chi}_{j}^{ \pm}}^{2}}{s+\Delta_{j}-w \cos \theta}-\frac{8 s m_{\tilde{\chi}_{j}^{ \pm}}^{2}}{\left(s+\Delta_{j}-w \cos \theta\right)^{2}}-\frac{3 s+3 \Delta_{j}+w \cos \theta}{2 s}\right\},
$$

where

$$
w \equiv \sqrt{s-\left(m_{\tilde{\chi}_{j}^{ \pm}}+m_{\tilde{\nu}_{e}}\right)^{2}} \sqrt{s-\left(m_{\tilde{\chi}_{j}^{ \pm}}-m_{\tilde{\nu}_{e}}\right)^{2}} \quad \text { and } \quad \Delta_{j} \equiv m_{\tilde{\chi}_{j}^{ \pm}}^{2}-m_{\tilde{\nu}_{e}}^{2} .
$$


Similarly for the total cross section for circularly polarized photons we obtain

$$
\sigma=\sigma^{0}\left(1-\lambda_{e}\right)\left(1+\lambda_{\gamma} A_{c}\right)
$$

with the unpolarized cross section

$$
\sigma^{0}=\frac{\left|V_{j 1}\right|^{2} \alpha^{2} \pi}{4 \sin ^{2} \theta_{W}} \frac{w}{s^{3}}\left\{\frac{2 s\left(s+2 \Delta_{j}\right)+4 \Delta_{j}^{2}}{w} \ln \frac{s+\Delta_{j}+w}{s+\Delta_{j}-w}-3 s-7 \Delta_{j}\right\} .
$$

The circular polarization asymmetry is given by

$$
A_{c} \sigma^{0}=\frac{\left|V_{j 1}\right|^{2} \alpha^{2} \pi}{4 \sin ^{2} \theta_{W}} \frac{w}{s^{3}}\left\{\frac{2 s\left(s+2 \Delta_{j}+2 m_{\tilde{\chi}_{j}^{ \pm}}^{2}\right)}{w} \ln \frac{s+\Delta_{j}+w}{s+\Delta_{j}-w}-7 s-3 \Delta_{j}\right\} .
$$

For linearly polarized photons the differential cross section shows an azimuthal $\cos 2 \phi$ dependence:

$$
\frac{d \sigma}{d \Omega}=\frac{d \sigma^{0}}{d \Omega}\left(1+a_{l} \cos 2 \phi\right)
$$

with

$$
\begin{aligned}
a_{l} \frac{d \sigma^{0}}{d \Omega} & =\frac{1}{2}\left(\frac{d \sigma_{\|}}{d \Omega}-\frac{d \sigma_{\perp}}{d \Omega}\right)=\frac{d \sigma_{\|}}{d \Omega}-\frac{d \sigma^{0}}{d \Omega}=\frac{d \sigma^{0}}{d \Omega}-\frac{d \sigma_{\perp}}{d \Omega} \\
& =\frac{\left|V_{j 1}\right|^{2} \alpha^{2}}{4 \sin ^{2} \theta_{W}} \frac{w^{3}}{s^{3}} \frac{\Delta_{j} \sin ^{2} \theta}{\left(s+\Delta_{j}-w \cos \theta\right)^{2}} .
\end{aligned}
$$

In equ. (12) $\frac{d \sigma_{\|}}{d \Omega}$ and $\frac{d \sigma_{\perp}}{d \Omega}$ denote the cross sections for linear photon polarization with polarization vector $\boldsymbol{\epsilon}_{\|}=\mathbf{p} \times\left(\mathbf{p}^{\prime} \times \mathbf{p}\right) /\left|\mathbf{p} \times\left(\mathbf{p}^{\prime} \times \mathbf{p}\right)\right|$ in the scattering plane and with polarization vector $\boldsymbol{\epsilon}_{\perp}=\mathbf{p}^{\prime} \times \mathbf{p} /\left|\mathbf{p}^{\prime} \times \mathbf{p}\right|$ perpendicular to it, respectively.

Note that due to the factor $\Delta_{j}=m_{\tilde{\chi}_{j}^{ \pm}}^{2}-m_{\tilde{\nu}_{e}}^{2}$ the sign of the asymmetry $a_{l}$ and the azimuthal dependence of the cross section is determined by the chargino-sneutrino mass difference. Especially for an invisible sneutrino it is always positive.

The best source of high energy photon beams is that of Compton backscattered intense laser pulses off one of the beams of a linear collider in the $e^{+} e^{-}$or $e^{-} e^{-}$mode. For longitudinally polarized electrons with energy $E_{e}$ and helicity $\bar{\lambda}_{e}$ and circularly polarized laser photons with energy $E_{L}$ and helicity $\lambda_{L}$ the photon energy spectrum is given by [6 8, 11]

$$
P\left(y, \bar{\lambda}_{e}, \lambda_{L}\right)=\frac{1}{\sigma_{c}} \frac{2 \pi \alpha^{2}}{x m_{e}^{2}}\left[\frac{1}{1-y}+1-y-4 r(1-r)-\bar{\lambda}_{e} \lambda_{L} r x(2 r-1)(2-y)\right]
$$

with $y=E_{\gamma} / E_{e}$, where $E_{\gamma}$ is the energy of the high energy photons, $x \equiv \frac{4 E_{e} E_{L}}{m_{e}^{2}}$ and $r \equiv \frac{y}{x(1-y)}$. The total Compton cross section

$$
\sigma_{c}=\sigma_{c}^{0}+\bar{\lambda}_{e} \lambda_{L} \sigma_{c}^{1}
$$

with 


$$
\begin{gathered}
\sigma_{c}^{0}=\frac{\pi \alpha^{2}}{x m_{e}^{2}}\left[\left(2-\frac{8}{x}-\frac{16}{x^{2}}\right) \ln (x+1)+1+\frac{16}{x}-\frac{1}{(x+1)^{2}}\right], \\
\sigma_{c}^{1}=\frac{\pi \alpha^{2}}{x m_{e}^{2}}\left[\left(2+\frac{4}{x}\right) \ln (x+1)-5+\frac{2}{x+1}-\frac{1}{(x+1)^{2}}\right]
\end{gathered}
$$

normalizes the distribution to $\int P(y) d y=1$. The fraction $y=E_{\gamma} / E_{e}$ of the photon and $e^{ \pm}$ beam energies varies in the range

$$
0 \leq y \leq \frac{x}{x+1} \quad \text { with } \quad x<2(1+\sqrt{2}) \approx 4.83
$$

For higher values of $x$ the conversion efficiency would be drastically reduced by production of $e^{+} e^{-}$pairs in collisions of high energy photons and laser photons.

Polarizing the electrons and/or the laser photons results in polarized backscattered photons with mean helicity

$$
\lambda\left(y, \bar{\lambda}_{e}, \lambda_{L}\right)=\frac{\lambda_{L}(1-2 r)\left(1-y+\frac{1}{1-y}\right)+\bar{\lambda}_{e} r x\left[1+(1-y)(1-2 r)^{2}\right]}{\frac{1}{1-y}+1-y-4 r(1-r)-\bar{\lambda}_{e} \lambda_{L} r x(2 r-1)(2-y)} .
$$

If the laser light has linear polarization $P_{t L}$ then for unpolarized electrons the high energy photons are linearly polarized in the same direction as the laser light. Their degree of polarization is

$$
P_{t}(y)=\frac{2 r^{2} P_{t L}}{\frac{1}{1-y}+1-y-4 r(1-r)}
$$

and the energy spectrum is given by Equ. (13) with $\bar{\lambda}_{e}=\lambda_{L}=0$.

To obtain the total cross section $\sigma_{e e}$ and the polarization asymmetry $A_{e e}$ in the laboratory frame we fold the $e \gamma$-cross section equ. (8) with the energy distribution equ. (13):

$$
\sigma_{e e}\left(s_{e e}, \bar{\lambda}_{e}, \lambda_{L}\right)=\int d y P\left(y, \bar{\lambda}_{e}, \lambda_{L}\right) \sigma\left(s_{e \gamma}, y, \bar{\lambda}_{e}, \lambda_{L}\right)
$$

with

$$
\left.\sigma\left(s_{e \gamma}, y, \bar{\lambda}_{e}, \lambda_{L}\right)=\sigma^{0}\left(s_{e \gamma}\right)\left(1-\lambda_{e}\right)\left\{1+\lambda\left(y, \bar{\lambda}_{e}, \lambda_{L}\right)\right) A_{c}\left(s_{e \gamma}\right)\right\}
$$

and

$$
s_{e \gamma}=y s_{e e} .
$$

Then the polarization asymmetry of the convoluted cross section

$$
A_{e e}\left(\bar{\lambda}_{e}, \lambda_{L}\right)=\frac{\sigma_{e e}\left(s_{e e}, \bar{\lambda}_{e}, \lambda_{L}\right)-\sigma_{e e}\left(s_{e e},-\bar{\lambda}_{e},-\lambda_{L}\right)}{\sigma_{e e}\left(s_{e e}, \bar{\lambda}_{e}, \lambda_{L}\right)+\sigma_{e e}\left(s_{e e},-\bar{\lambda}_{e},-\lambda_{L}\right)}
$$

becomes

$$
A_{e e}\left(\bar{\lambda}_{e}, \lambda_{L}\right)=\frac{\int d y P\left(y, \bar{\lambda}_{e}, \lambda_{L}\right) \lambda\left(y, \bar{\lambda}_{e}, \lambda_{L}\right) \sigma^{0}\left(y s_{e e}\right) A_{c}\left(y s_{e e}\right)}{\int d y P\left(y, \bar{\lambda}_{e}, \lambda_{L}\right) \sigma^{0}\left(y s_{e e}\right)}
$$




\section{NUMERICAL RESULTS AND DISCUSSION}

\section{A. Scenarios}

For the numerical calculations we have chosen three characteristic scenarios of gauginohiggsino mixing. Table $\mathbb{1}$ shows the masses of the charginos and the two lightest neutralinos and the mixing parameters $V_{i j}$ of the charginos [10] relevant for their coupling to electrons and sneutrinos. Also shown is the state of the lightest neutralino $\tilde{\chi}_{1}^{0}$ in the basis of the weak eigenstates $\left(\tilde{\gamma}, \tilde{Z}, \tilde{H}_{a}^{0}, \tilde{H}_{b}^{0}\right)$. Generally the masses and couplings of the charginos and neutralinos depend on the four parameters $M, M^{\prime}, \mu$ and $\tan \beta$ [10,12]. As usual $M^{\prime}$ is fixed by $M^{\prime} / M=5 / 3 \tan ^{2} \theta_{W}$ and since in most cases the dependence on the parameter $\tan \beta$ is rather weak, we have chosen $\tan \beta=2$. The parameters $M$ and $\mu$ have been chosen such, that in all three cases the mass of the lightest chargino $\tilde{\chi}_{1}^{ \pm}$is about $88 \mathrm{GeV}$. The main difference between the three scenarios is the state of the lightest neutralino. In scenario A the LSP is almost a photino, in scenario $\mathrm{C}$ it is nearly a pure higgsino and in scenario $\mathrm{B}$ it is a photino-zino-higgsino mixture.

Assuming renormalization group equations, the sfermion masses are related to the SUSY parameters by [13, 14

$$
m_{\tilde{f}_{L / R}}^{2}=m_{f}^{2}+m_{0}^{2}+C(\tilde{f}) M^{2} \pm m_{Z}^{2} \cos 2 \beta\left(T_{3 f}-e_{f} \sin ^{2} \theta_{W}\right)
$$

where $m_{0}$ is the common scalar mass at the unification point, $T_{3 f}$ and $e_{f}$ are the third component of the weak isospin and the charge of the corresponding left- or right-handed fermion, respectively, and $C\left(\tilde{l}_{R}\right) \approx 0.23, C\left(\tilde{l}_{L}\right)=C(\tilde{\nu}) \approx 0.79, C\left(\tilde{q}_{L}\right) \approx 10.8, C\left(\tilde{q}_{R}\right) \approx 10.1$.

Then for any value of $m_{0}$ there exist certain regions in the parameter space where the sneutrino is lighter than the lightest chargino $\tilde{\chi}_{1}^{ \pm}$and the second lightest neutralino $\tilde{\chi}_{2}^{0}$. In these regions only the invisible decay $\tilde{\nu}_{e} \rightarrow \nu_{e}+\tilde{\chi}_{1}^{0}$ is allowed leading to one-sided events with the detailed signatures determined by the branching ratios for the chargino decay channels.

Fig. 2 shows these regions of an invisible sneutrino for the two values $m_{0}=50 \mathrm{GeV}$ and $m_{0}=100 \mathrm{GeV}$ of the scalar mass and $\tan \beta=2$. For small values of $m_{0}$ and especially for $\mu<0$ the sneutrino will decay invisibly in a considerable domain of the $M$ - $\mu$ plane. With increasing $m_{0}$ these regions are shifted to unphysical large values of $M$ and $\mu$. Also shown are the parameter space excluded by the chargino mass bound $65 \mathrm{GeV}$ recently published by LEP1.5 [15] and the position of the scenarios A, B and C. For $m_{0} \lesssim 76.8 \mathrm{GeV}$ the sneutrino is invisible in scenario A.

\section{B. Results for monochromatic photons}

In the following cross sections are given for unpolarized electrons. Due to the factor $\left(1-\lambda_{e}\right)$ they will be enhanced by a factor 2 for left-polarized electron beams $\left(\lambda_{e}=-1\right)$.

Fig. 3 displays the total cross sections for unpolarized electrons and for different photon polarizations for the production of the light chargino $\tilde{\chi}_{1}^{-}$in scenario A for $m_{0}=50 \mathrm{GeV} . \sigma_{+}$ and $\sigma_{-}$denote the cross sections for photon helicity $\lambda_{\gamma}= \pm 1$, respectively. For comparison we have added the $\theta$-integrated cross sections for linearly polarized photons, multiplied by $2 \pi\left(\sigma_{\| / \perp} \equiv 2 \pi d \sigma_{\| / \perp} / d \phi\right)$. For CMS-energies $\sqrt{s} \lesssim 400 \mathrm{GeV}$ the cross section is the highest 
for $\lambda_{\gamma}=-1$ whereas for $\sqrt{s} \gtrsim 400 \mathrm{GeV}$ it is the highest for $\lambda_{\gamma}=+1$. Since in scenario A the cross section for the heavy chargino is smaller than $0.002 \mathrm{pb}$ we omit the graph for $\tilde{\chi}_{2}^{-}$-production.

In scenario $\mathrm{B}$ the cross sections for $\tilde{\chi}_{1}^{-}$-production (fig. 4 ) is of the same order of magnitude as in scenario A. Again for $\sqrt{s} \lesssim 450 \mathrm{GeV}$ it is the largest for $\lambda_{\gamma}=-1$ whereas for higher energies $\sqrt{s} \gtrsim 450 \mathrm{GeV} \lambda_{\gamma}=+1$ leads to larger values. Here due to the larger wino component of the heavy chargino the cross section for $\tilde{\chi}_{2}^{-}$is two orders of magnitude higher than in scenario A.

In scenario $\mathrm{C}$ (fig. 5) finally the large wino component of the heavy chargino compensates the mass difference between the heavy and the light one so that apart from the different thresholds both cross sections for $e^{-} \gamma \rightarrow \tilde{\nu}_{e} \tilde{\chi}_{1}^{-}$and $e^{-} \gamma \rightarrow \tilde{\nu}_{e} \tilde{\chi}_{2}^{-}$are of the same order. For high energies the cross section for the heavy chargino even dominates.

Varying the scalar mass $m_{0}$ from $m_{0}=50 \mathrm{GeV}$ to $m_{0}=200 \mathrm{GeV}$ increases the sneutrino mass without changing the sneutrino couplings. Apart from the higher threshold the cross sections are from the same order of magnitude and their energy dependence is very similar for both values of $m_{0}$. Even in scenario A with very different sneutrino mass for $m_{0}=50 \mathrm{GeV}$ $\left(m_{\tilde{\nu}_{e}}=65.1 \mathrm{GeV}\right)$ and $m_{0}=200 \mathrm{GeV}\left(m_{\tilde{\nu}_{e}}=204.3 \mathrm{GeV}\right)$ the maximum cross section for $\tilde{\chi}_{1}^{-}$-production with unpolarized photons is reduced by only $50 \%$ and that for circularly polarized photons with $\lambda_{\gamma}=-1$ by only $35 \%$. For scenarios $\mathrm{B}$ and $\mathrm{C}$ and for the heavy chargino the reduction of the maximum cross section is always less than $50 \%$. Therefore we renounce the graphs for the case $m_{0}=200 \mathrm{GeV}$.

Due to the factor $\Delta_{j}=m_{\tilde{\chi}_{j}^{ \pm}}^{2}-m_{\tilde{\nu}_{e}}^{2}$ in equ. (12) in scenario A for $e^{-} \gamma \rightarrow \tilde{\chi}_{1}^{-} \tilde{\nu}_{e}$ and in scenarios $\mathrm{B}$ and $\mathrm{C}$ for $e^{-} \gamma \rightarrow \tilde{\chi}_{2}^{-} \tilde{\nu}_{e}$ the asymmetry for linearly polarized photons changes sign when we turn from $m_{0}=50 \mathrm{GeV}$ to $m_{0}=200 \mathrm{GeV}$. Quite generally, however, the nature of the chargino is by far more crucial than the mass of the sneutrino.

To illustrate the influence of wino-higgsino mixing we give in fig. 6 the contour lines of the cross sections for $e^{-} \gamma \rightarrow \tilde{\chi}_{1}^{-} \tilde{\nu}_{e}$ and $e^{-} \gamma \rightarrow \tilde{\chi}_{2}^{-} \tilde{\nu}_{e}$ for unpolarized photons and both values of $m_{0}$. We have marked in fig. 6 (a) $\left(m_{0}=50 \mathrm{GeV}\right)$ the regions of an invisible sneutrino while for $m_{0}=200 \mathrm{GeV}$ they are shifted to large values of $M$ and $\mu(M \gtrsim 410 \mathrm{GeV}$, $\mu \lesssim-500 \mathrm{GeV}$ for $\mu<0$ and $M \gtrsim 420 \mathrm{GeV}, \mu \gtrsim 740 \mathrm{GeV}$ for $\mu>0$ ).

The circular polarization asymmetry $A_{c}$ of the total cross sections is depicted in fig. 7 for scenario A with $m_{0}=50 \mathrm{GeV}$. Apart from a somewhat different threshold behavior the magnitude as well as the energy dependence is very similar for $m_{0}=200 \mathrm{GeV}$. In both cases for the light chargino it is varying between $A_{c}=-1$ and $A_{c}=+0.4$ between threshold and $\sqrt{s}=1000 \mathrm{GeV}$. For the heavy chargino the circular polarization asymmetry $A_{c},<0$ in this energy region, changes sign at $\sqrt{s} \approx 1000 \mathrm{GeV}\left(A_{c} \rightarrow 1\right.$ for $\left.\sqrt{s} \rightarrow \infty\right)$.

Contrary to the circular polarization asymmetry the linear polarization asymmetry $a_{l}$ for scenario $\mathrm{A}$ is very different for $m_{0}=50 \mathrm{GeV}$ and $m_{0}=200 \mathrm{GeV}$ (fig. 8). Due to the factor $\Delta_{j}$ in equ. (12) it is positive for the heavy chargino for both values of $m_{0}$ whereas for the light chargino $a_{l}>0$ if the sneutrino decays invisible $\left(m_{0}=50 \mathrm{GeV}\right)$ and $a_{l}<0$ for $m_{0}=200 \mathrm{GeV}$ when it is heavier than the light chargino $\left(a_{l} \rightarrow 0\right.$ for $\left.\sqrt{s} \rightarrow \infty\right)$.

We omit the graphs for the polarization asymmetries for scenarios B and C. Those for the circular polarization asymmetry are practically identical with those for scenario A apart from different threshold energies. For the light chargino the behavior of the linear polarization 
asymmetry $a_{l}$ differs inappreciable from that in scenario A for the case of a visible sneutrino $\left(m_{0}=200 \mathrm{GeV}\right)$. For the heavy chargino the linear polarization asymmetry for $m_{0}=50 \mathrm{GeV}$ is less than $15 \%$ for scenario $\mathrm{B}$, less than $10 \%$ for scenario $\mathrm{C}$ and for $m_{0}=200 \mathrm{GeV}$ it is less than $5 \%$ in scenario C. Because of $m_{\tilde{\nu}_{e}} \approx m_{\tilde{\chi}_{2}^{ \pm}}$in scenario B with $m_{0}=200 \mathrm{GeV}$ the linear polarization asymmetry $a_{l}$ is very small $(<1 \%)$.

\section{Results for an ee collider}

Before entering the discussion of polarization effects we show in fig. 9 the contour lines of the convoluted cross sections for unpolarized beams with CMS-energy $\sqrt{s_{e e}}=500 \mathrm{GeV}$. Since the reaction is particularly suitable for the detection of invisible sneutrinos we have chosen $m_{0}=50 \mathrm{GeV}$ for the scalar mass. Then in a large region of the parameter space (see fig. 6(a)) only the invisible decay of the sneutrino into the LSP is kinematically allowed. As can be inferred from fig. 6 increasing $m_{0}$ will shift the threshold to higher energies and reduce the convoluted cross sections between $30 \%$ and $50 \%$ for $m_{0}=200 \mathrm{GeV}$.

Corresponding to the energy $\frac{x}{x+1} E_{e} \sim 0.83 E_{e}$ of the hardest photons the kinematical accessible $M-\mu$ region is somewhat smaller as in fig. 6. This results in a reduction of the cross sections for $e e \rightarrow \tilde{\nu}_{e} \tilde{\chi}_{j}^{-}$and $\sqrt{s_{e e}}=500 \mathrm{GeV}$ compared to those for $e \gamma \rightarrow \tilde{\nu}_{e} \tilde{\chi}_{j}^{-}$and $\sqrt{s_{e \gamma}}=500 \mathrm{GeV}$ for scenarios close by the kinematical boundary in the $M-\mu$ plane. Thus the cross section for production of heavy charginos in scenario $\mathrm{C}$ is reduced by more than one order of magnitude while those for the light chargino are of the same order in all three scenarios.

Fig. 10 shows the energy dependence of the convoluted cross section for scenario A with $m_{0}=50 \mathrm{GeV}$. For comparison we have included also that for $e^{-} \gamma \rightarrow \tilde{\chi}_{1}^{-} \tilde{\nu}_{e}$ for unpolarized photons. Notice that due to the energy distribution of the backscattered photons for energies $\sqrt{s_{e e}}$ high enough the convoluted cross section is larger than that for $e^{-} \gamma \rightarrow \tilde{\chi}_{1}^{-} \tilde{\nu}_{e}$ for the same $e \gamma$-energy $\sqrt{s_{e \gamma}}=\sqrt{s_{e e}}$. The background processes displayed in fig. 10 will be discussed in the next section.

Both the energy spectrum and the mean helicity (equs. (13) and (18)) of the high energy photons sensibly depend on the helicity $\lambda_{L}$ of the laser photons and the helicity $\bar{\lambda}_{e}$ of the converted electrons [7]. We therefore discuss in the following the asymmetries $A_{e e}\left(\bar{\lambda}_{e}, \lambda_{L}\right)$ of the convoluted cross sections for the production of the light chargino in scenario A with $m_{0}=50 \mathrm{GeV}$ for several polarization configurations $\left(\bar{\lambda}_{e}, \lambda_{L}\right)$.

Since due to missing energy it is impossible to reconstruct from the momenta of the decay products of the supersymmetric particles the production plane we renounce the discussion of convoluted cross sections for linearly polarized photons. For a significant discussion one has to include the decays of the chargino and to study, e.g., the linear polarization asymmetry of the observable leptons. This is, however, beyond the scope of our study.

Generally one obtains large negative polarization asymmetries for lower energies near the threshold and smaller positive asymmetries for higher energies. The energy region where the asymmetry changes sign and is therefore small depends on the polarization configuration. If only the electron beam is polarized (fig. 11(a)), the asymmetry attains $-65 \%$ for $\sqrt{s_{e e}}=$ $200 \mathrm{GeV}$ and is of the order of $+15 \%$ for the highest energy $\sqrt{s_{e e}}=1000 \mathrm{GeV}$. In the energy region around $500 \mathrm{GeV}$ the asymmetry is small. Apart from shifting the energy region 
where the asymmetry changes the sign the situation is very similar if additionally the laser photons are polarized (figs. 11(b), (c)). In fig. 11(b) the steep descent of the asymmetry just beyond threshold originates from the special energy dependence of the mean helicity of the backscattered photons. For $\bar{\lambda}_{e}=\lambda_{L}=+1$ is $\lambda\left(y, \bar{\lambda}_{e}, \lambda_{L}\right)=+1$ over almost the whole spectrum with a steep descent to $\lambda\left(y, \bar{\lambda}_{e}, \lambda_{L}\right)=-1$ close to the high energy end [7]. With exception of the region very close to threshold the asymmetry for unpolarized electrons and polarized photons (fig. [11(d)) is substantially smaller with values between $-20 \%$ for $\sqrt{s_{e e}}=200 \mathrm{GeV}$ and $+20 \%$ for $\sqrt{s_{e e}}=340 \mathrm{GeV}$.

The discussion of the polarization asymmetries for the background processes displayed in fig. 11 shall be postponed to the following section.

\section{SIGNATURES AND BACKGROUND}

For the feasibility of the detection of SUSY signals the decay patterns of the produced particles are as crucial as their production cross sections. Our investigations should therefore be completed by an analysis of the decay channels and a study of the standard model background. For the present we shall however restrict ourselves to some remarks emphasizing the usefulness of polarized beams. For scenario A with $m_{0}=200 \mathrm{GeV}$ and $m_{\tilde{\nu}_{e}}=204.3 \mathrm{GeV}$ as well as for scenarios $\mathrm{B}$ and $\mathrm{C}$ with heavy sneutrinos the dominating signature with branching ratio between $35 \%$ and $40 \%$ are four jets accompanied by one electron (or one positron) via

$$
\begin{aligned}
e^{-}+\gamma \longrightarrow \tilde{\nu}_{e}+\tilde{\chi}_{1}^{-} \\
\stackrel{\llcorner}{\longleftrightarrow} \bar{u}+d+\tilde{\chi}_{1}^{0} \\
\stackrel{\stackrel{L}{\longrightarrow}+\tilde{\chi}_{1}^{+}}{\longmapsto} u+\bar{d}+\tilde{\chi}_{1}^{0} .
\end{aligned}
$$

The decay rates and the branching ratios of the supersymmetric particles have been calculated with the formulae given in refs. [16,17.

The standard model background for this signature resulting from higher-order processes is expected to be rather small. Since, however, a heavy sneutrino decaying visibly should also be observable at an $e^{+} e^{-}$collider we shall not further consider this case. We focus on the particular interesting case of associated production of a light invisible sneutrino and the light chargino realized in scenario A with $m_{0}=50 \mathrm{GeV}$. In this case the chargino almost exclusively decays into the invisible $\tilde{\nu}_{e}$ and one electron so that for $\sqrt{s_{e e}}=500 \mathrm{GeV}$ the cross section for $e^{-} \gamma \rightarrow e^{-}+\not E$ attains values between $1 \mathrm{pb}$ and $2 \mathrm{pb}$. On account of the small cross section contributions from the production and decay of the heavy chargino they are negligible. For this case the $e \gamma$ option seems to be superior to the radiative process $e^{+} e^{-} \rightarrow \tilde{\nu} \overline{\tilde{\nu}} \gamma$ with cross sections at least two orders of magnitude smaller [4].

The most important background to the signal arises from the two processes $e^{-} \gamma \rightarrow W^{-} \nu_{e}$ and $e^{-} \gamma \rightarrow e^{-} Z$. The cross sections for this processes shown in fig. 10 are discussed in detail in [18 21] and yield values of $30 \mathrm{pb}$ and $8 \mathrm{pb}$ for $\sqrt{s_{e e}}=500 \mathrm{GeV}$, respectively. This seems very high but the background is reduced to $\sigma\left(e \gamma \rightarrow\left(W \nu_{e}, e Z\right) \rightarrow 1 e^{-}+\not E\right) \approx 9$ pb for the $\left(1 e^{-}+\not E\right)$-signature. Furthermore one expects, that similar as for the process $e \gamma \rightarrow \tilde{e} \tilde{\chi}_{j}^{0}$ 
the supersymmetric signal can be enhanced relative to the SM background by cuts on the rapidity and transverse momentum of the final state electron [8].

Although the contribution from $e^{-} \gamma \rightarrow e^{-} Z$ is rapidly decreasing with increasing energy one should note that it is more important for the convoluted cross sections than for the nonconvoluted ones, compared for the same CMS energy $\sqrt{s_{e e}}$ and $\sqrt{s_{e \gamma}}$, respectively; e.g., one finds $\sigma(e \gamma \rightarrow e Z) \sim 3 \mathrm{pb}(0.8 \mathrm{pb})$ for $\sqrt{s_{e \gamma}}=500 \mathrm{GeV}(1000 \mathrm{GeV})$ whereas due to folding with the photon spectrum $\sigma(e e \rightarrow e \gamma \rightarrow e Z) \sim 8 \mathrm{pb}(3 \mathrm{pb})$ for $\sqrt{s_{e e}}=500 \mathrm{GeV}$ $(1000 \mathrm{GeV})$.

The elimination of the SM background can considerably be facilitated by a polarized electron beam (fig. 11(a)). In most of the energy region between threshold and $1 \mathrm{TeV}$ the polarization asymmetries of the background processes are considerably smaller or even have different sign than that for the supersymmetric process. Comparison of fig. 11.(a) with figs. 11(b), (c) demonstrates that additional polarization of the laser photons would not substantially improve the situation. Thus combined with cuts in rapidity and transverse momentum the polarization asymmetries for a polarized electron beam will improve chances for elimination of the SM background.

Beyond associated production of an invisible sneutrino and the light chargino the supersymmetric process $e^{-} \gamma \rightarrow \tilde{e}^{-} \tilde{\chi}_{1}^{0} \rightarrow e^{-} \tilde{\chi}_{1}^{0} \tilde{\chi}_{1}^{0}$ will contribute to the single electron signal. This is on the one hand an additional background for the process under study, on the other hand it will enhance the supersymmetric signal relative to the SM background. We shall leave the study of this process to further investigations.

\section{SUMMARY}

We have calculated the cross sections and polarization asymmetries for the reaction $e^{-} \gamma \rightarrow \tilde{\chi}_{j}^{-} \tilde{\nu}_{e}$. The high energy photons can be generated in the $e \gamma$ collision mode by Compton backscattering of intense laser pulses off one of the beams of an $e^{+} e^{-}$or $e^{-} e^{-}$ linear collider. To obtain the cross sections and polarization asymmetries in the laboratory frame they must be convoluted with the Compton energy spectrum.

We have chosen three characteristic scenarios of gaugino-higgsino mixing for the numerical calculations. The cross sections for the production of the light chargino are of the order of $1 \mathrm{pb}$ in a considerably domain of the parameter space.

For sneutrinos decaying invisibly into a neutrino and the LSP the lighter chargino decays through a dominant two-body decay channel nearly exclusively in a sneutrino and an electron yielding a clear signature. Therefore the reaction $e^{-} \gamma \rightarrow \tilde{\chi}_{1}^{-} \tilde{\nu}_{e}$ is suitable to search for such invisible sneutrinos because here the cross sections are two orders of magnitude higher than for the radiative production of the sneutrinos [1].

The polarization asymmetries of the SM background processes $e \gamma \rightarrow W \nu_{e}$, e $\rightarrow \rightarrow e Z$ are smaller or have opposite sign for most of the energy region. So using polarized beams combined with cuts in rapidity and transverse momentum can considerably enhance the signal-to-background ratio. 


\section{ACKNOWLEDGMENTS}

We would like to thank F. Franke for many helpful comments on the manuscript. This work was supported by the Deutsche Forschungsgemeinschaft under contract no. FR 1064/2-1. 


\section{REFERENCES}

[1] A. Datta, M. Guchait, M. Drees, Z. Phys. C 69, 347 (1996).

[2] The ALEPH Collaboration, D. Decamp et al., Phys. Rep. 216, 253 (1992).

[3] G. Giacomelli, P. Giacomelli, Riv. Nuovo Cim. 16(3), 1 (1993).

[4] F. Franke, H. Fraas, Phys. Rev. D 49, 3126 (1994).

[5] H. E. Haber, G. L. Kane, Phys. Rep. 117, 75 (1985).

[6] I. F. Ginzburg, G. L. Kotkin, S. L. Panfil, V. G. Serbo, V. I. Telnov, Nucl. Instrum. Methods Phys. Res. A 219, 5 (1984).

[7] D. L. Borden, D. A. Bauer, D. O. Caldwell, Report No. SLAC-PUB-5715, 1992 (unpublished); Report No. UCSB-HEP-92-01, 1992 (unpublished); Phys. Rev. D 48, 4018 (1993).

[8] F. Cuypers, G. J. van Oldenborgh, R. Rückl, in $e^{+} e^{-}$Collisions at 500 GeV: The Physics Potential, Part C, Proceedings of the Workshop, Munich, Annecy, Hamburg, Germany, 1993, edited by P. M. Zerwas (DESY Report No. 93-123C, Hamburg, 1993), p. 475.

[9] J. A. Grifols, R. Pascual, Phys. Lett. 135B, 319 (1984); 142B, 455(E) (1984).

[10] A. Bartl, H. Fraas, W. Majerotto, B. Mösslacher, Z. Phys. C 55, 257 (1992).

[11] F. Cuypers, G. J. van Oldenborgh, R. Rückl, Nucl. Phys. B383, 45 (1992).

[12] A. Bartl, H. Fraas, W. Majerotto, N. Oshimo, Phys. Rev. D 40, 1594 (1989).

[13] A. Bartl, M. Dittmar, W. Majerotto, in $e^{+} e^{-}$Collisions at 500 GeV: The Physics Potential, Part B, Proceedings of the Workshop, Munich, Annecy, Hamburg, Germany, 1991, edited by P. M. Zerwas (DESY Report No. 92-123B, Hamburg, 1992), p. 603.

[14] L. J. Hall, J. Polchinski, Phys. Lett. 152B, 335 (1985).

[15] The ALEPH Collaboration, D. Buskulic et al., Phys. Lett. B 373, 246 (1996);

The L3 Collaboration, M. Acciarri et al., Phys. Lett. B 377, 289 (1996);

The OPAL Collaboration, G. Alexander et al., Phys. Lett. B 377, 181 (1996).

[16] T. Wöhrmann, PhD thesis, University of Würzburg 1993; T. Wöhrmann, H. Fraas, Phys. Rev. D 52, 78 (1995).

[17] A. Bartl, H. Fraas, W. Majerotto, Z. Phys. C 41, 475 (1988).

[18] A. Denner, S. Dittmaier, Nucl. Phys. B398, 239 (1993); B398, 265 (1993).

[19] M. Baillargeon, G. Bélanger, F. Boudjema, in Two-Photon Physics. From DAФNE to LEP200 and Beyond, Paris, France (World Scientific, Singapore, 1994), p. 267.

[20] G. Bélanger, F. Boudjema, in $e^{+} e^{-}$Collisions at 500 GeV: The Physics Potential, Part $B$, Proceedings of the Workshop, Munich, Annecy, Hamburg, Germany, 1991, edited by P. M. Zerwas (DESY Report No. 92-123B, Hamburg, 1992), p. 783.

[21] F. M. Renard, Z. Phys. C 14, 209 (1982). 


\section{FIGURES}

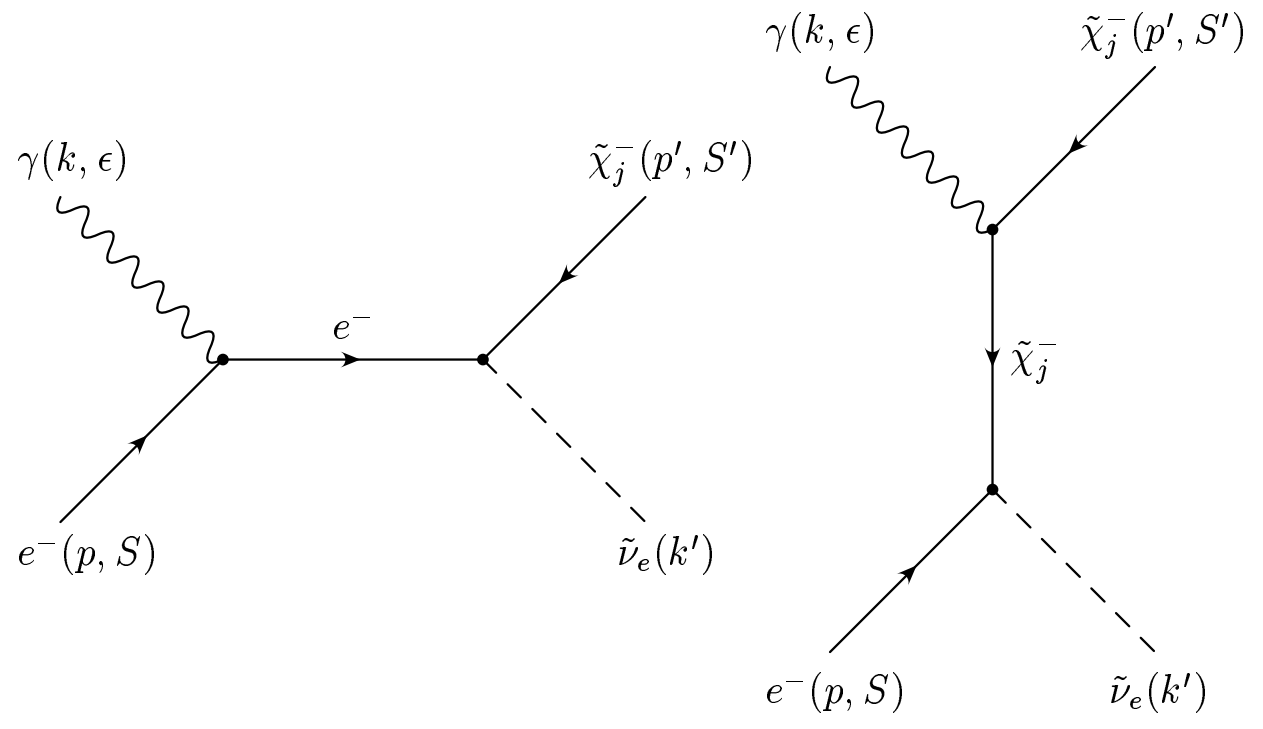

FIG. 1. Feynman graphs for $e^{-}+\gamma \rightarrow \tilde{\chi}_{j}^{-}+\tilde{\nu}_{e}$. 


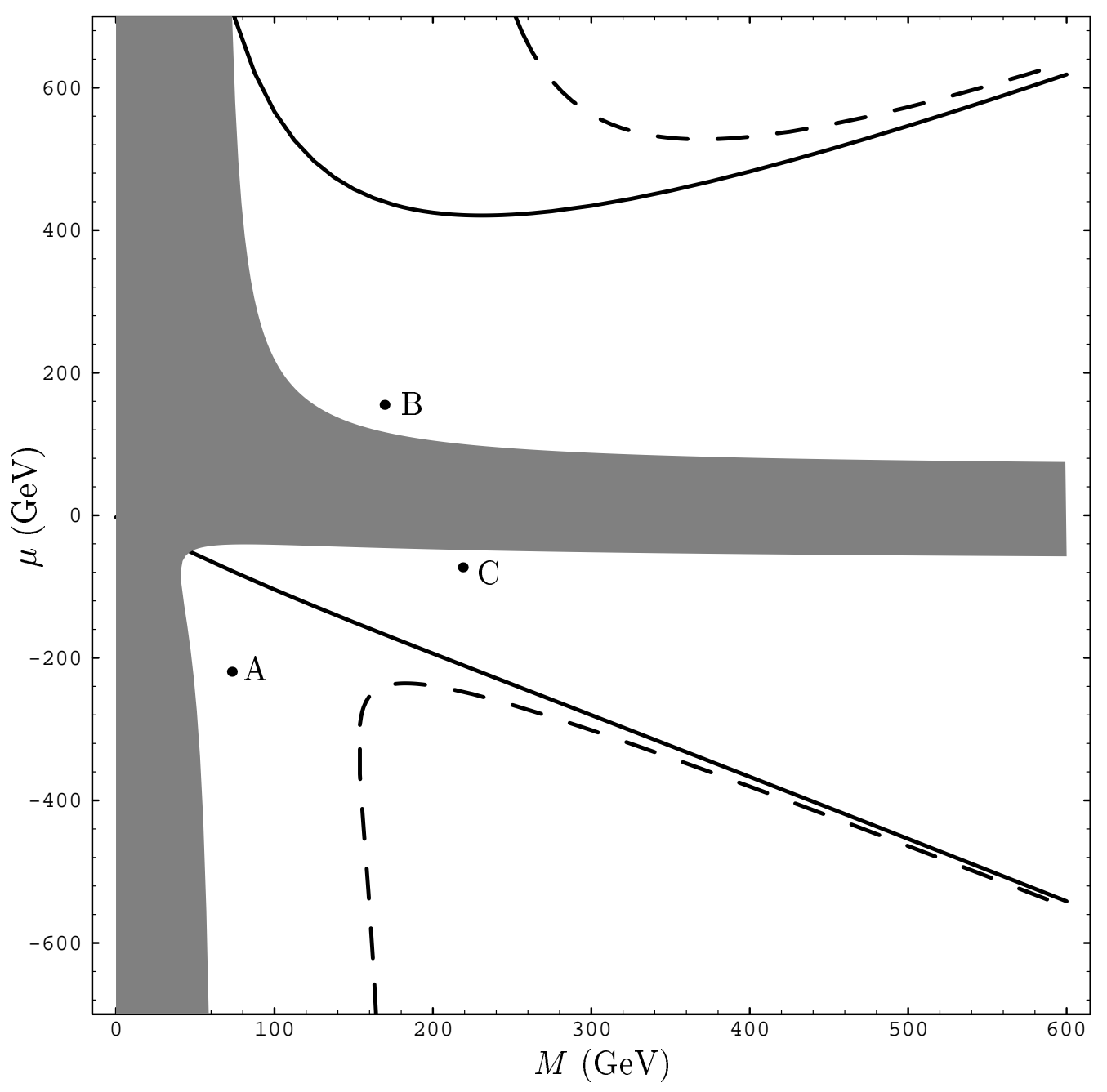

FIG. 2. Regions of an invisible sneutrino in the $M-\mu$ parameter space for the two values $m_{0}=50 \mathrm{GeV}$ (solid line) and $m_{0}=100 \mathrm{GeV}$ (dashed line) of the scalar mass and $\tan \beta=2$. The sneutrinos are invisible above the upper lines and below the lower lines, respectively. Also shown are the experimentally excluded parameter space (shaded area) and the position of the three scenarios A, B and C. 


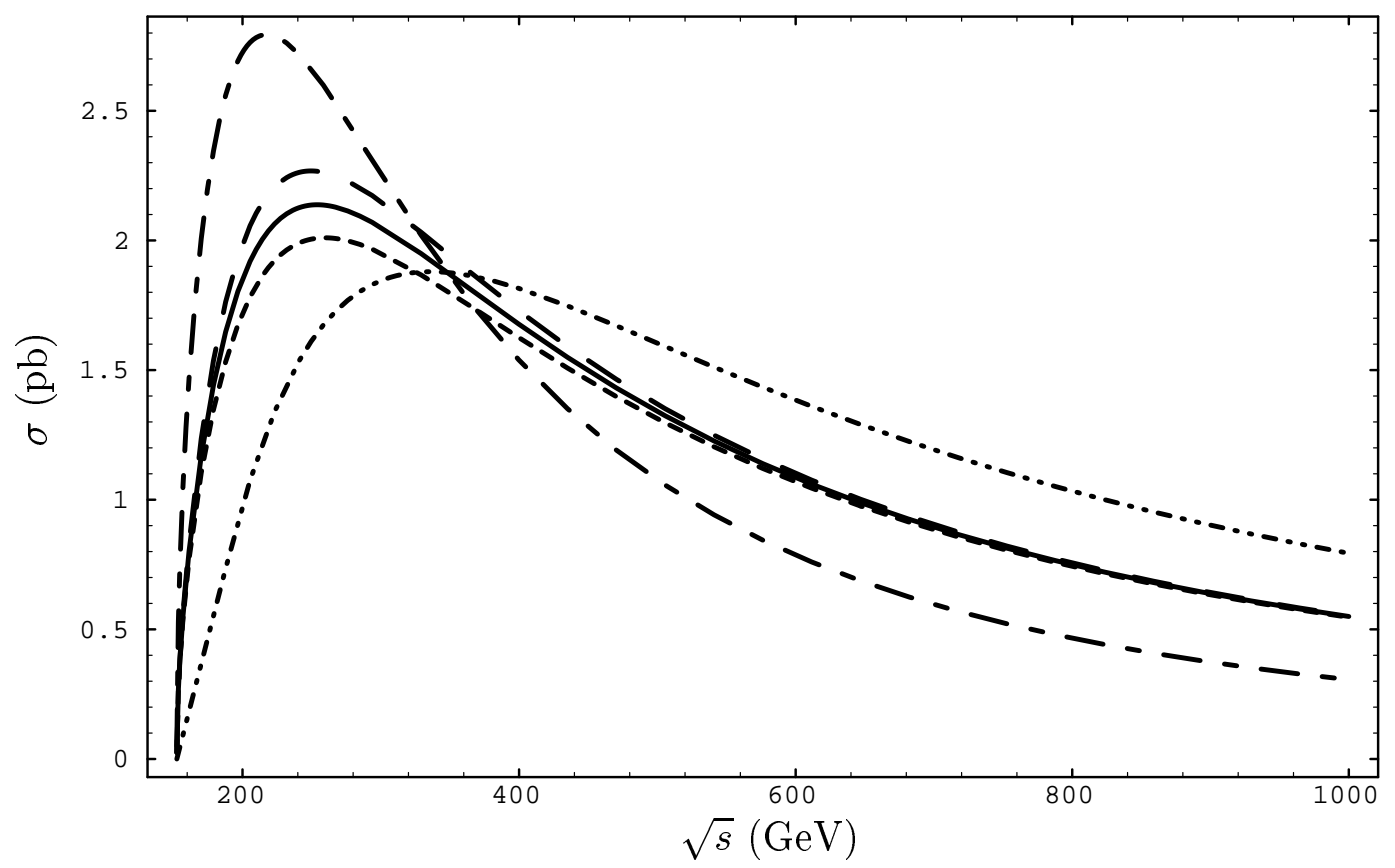

FIG. 3. Total cross sections $\sigma\left(e^{-} \gamma \rightarrow \tilde{\chi}_{1}^{-} \tilde{\nu}_{e}\right)$ in scenario A for $m_{0}=50 \mathrm{GeV}: \sigma^{0}(-(-)$, $\sigma_{\|}(--), \sigma_{\perp}(----), \sigma_{+}(-\cdots-), \sigma_{-}(---)$.

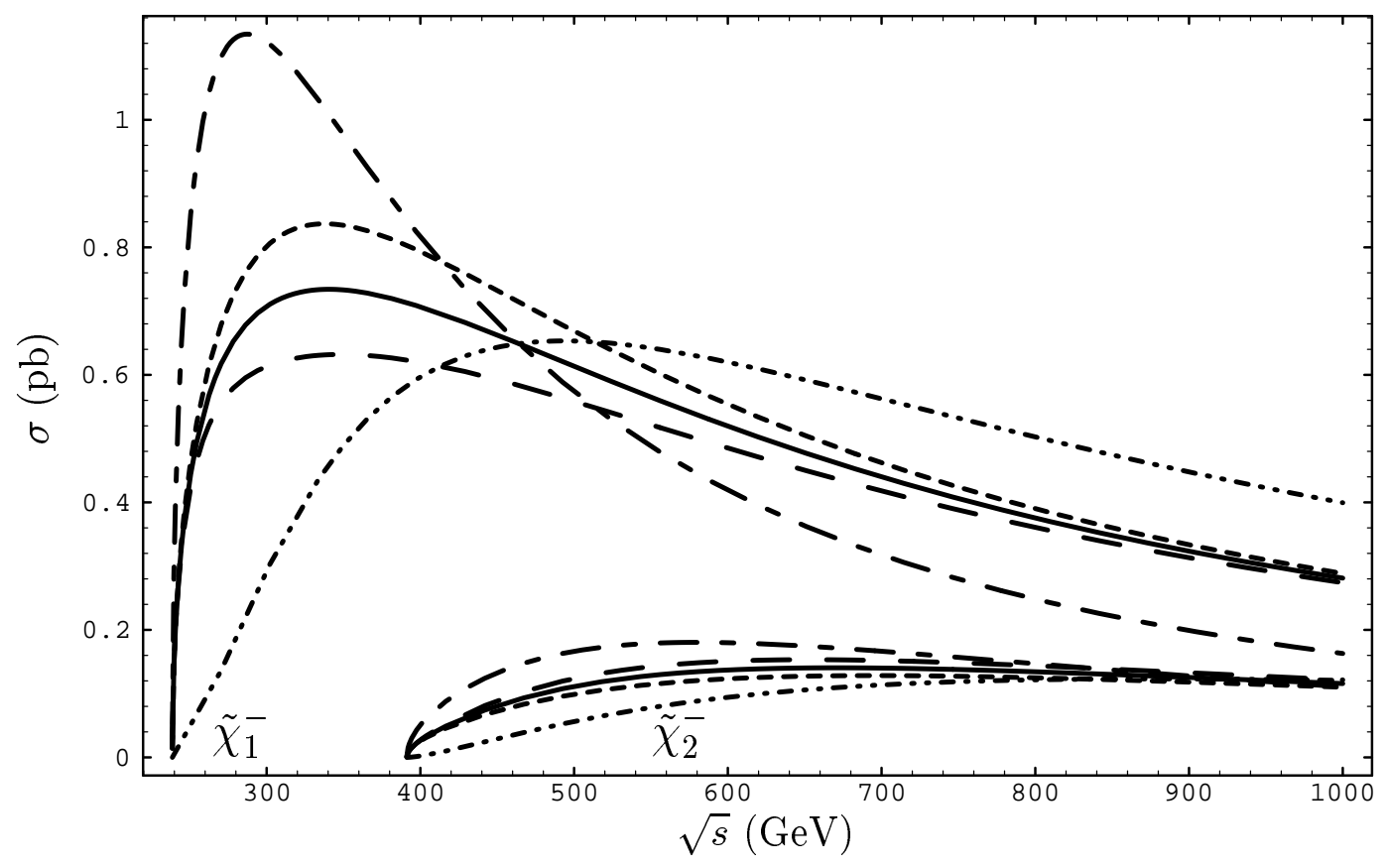

FIG. 4. Total cross sections $\sigma\left(e^{-} \gamma \rightarrow \tilde{\chi}_{1,2}^{-} \tilde{\nu}_{e}\right)$ in scenario B for $m_{0}=50 \mathrm{GeV}: \sigma^{0}(-(-)$, $\sigma_{\|}(--), \sigma_{\perp}(----), \sigma_{+}(-\cdots-), \sigma_{-}(---)$. 


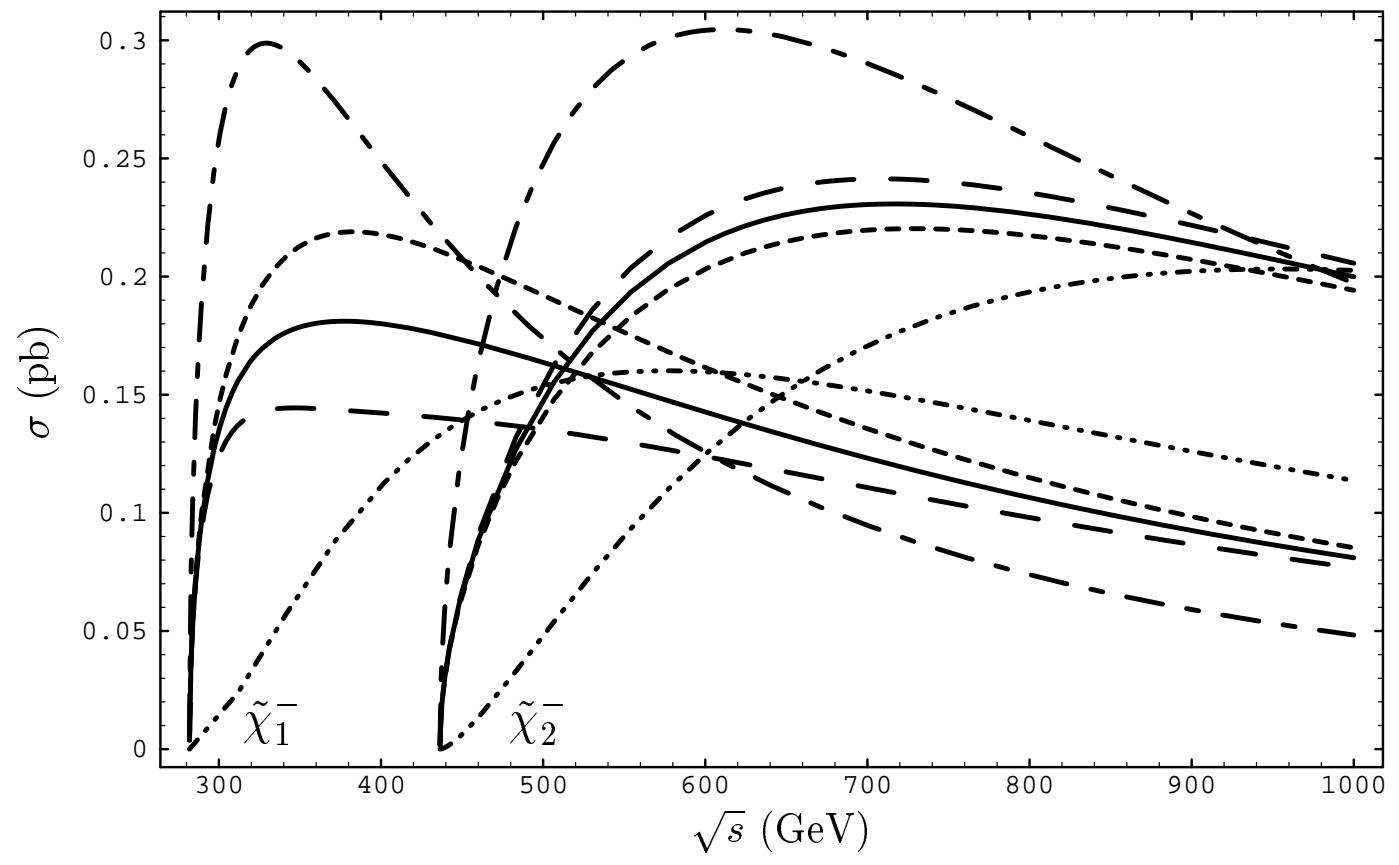

FIG. 5. Total cross sections $\sigma\left(e^{-} \gamma \rightarrow \tilde{\chi}_{1,2}^{-} \tilde{\nu}_{e}\right)$ in scenario $\mathrm{C}$ for $m_{0}=50 \mathrm{GeV}: \sigma^{0}(-\longrightarrow)$, $\sigma_{\|}(---), \sigma_{\perp}(----), \sigma_{+}(-\cdots-), \sigma_{-}(---)$. 

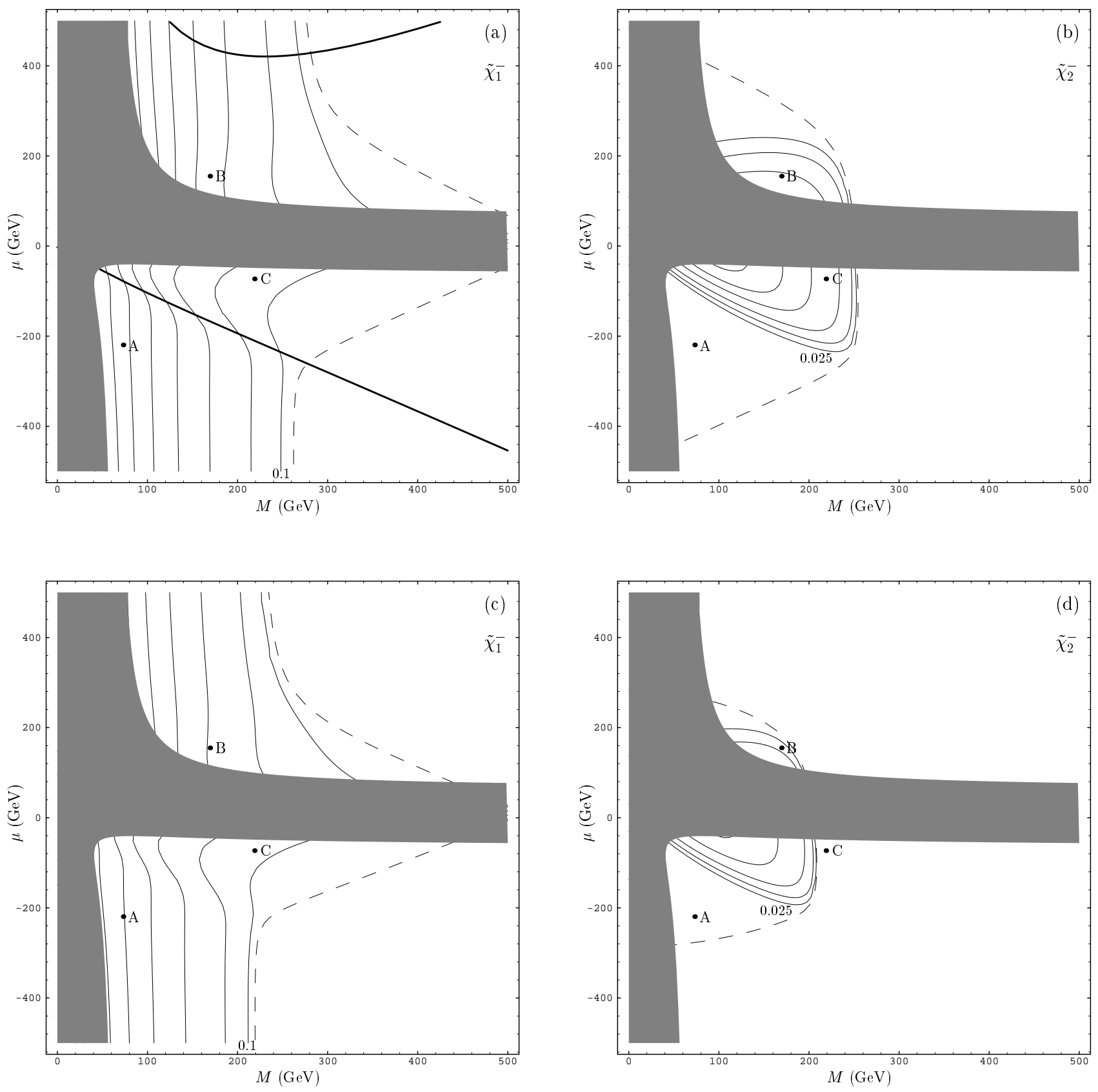

FIG. 6. Contours of the total cross section for $\sqrt{s}=500 \mathrm{GeV}$ and $\tan \beta=2$. The dashed lines mark the boundary $m_{\tilde{\chi}_{1,2}^{ \pm}}+m_{\tilde{\nu}_{e}}=500 \mathrm{GeV}$. The experimentally excluded domain is shaded. (a) Contours (0.1 pb, $0.25 \mathrm{pb}, 0.5 \mathrm{pb}, 0.75 \mathrm{~b}, 1.0 \mathrm{pb}, 1.25 \mathrm{pb}$ and $1.5 \mathrm{pb})$ of $\sigma\left(e^{-} \gamma \rightarrow \tilde{\chi}_{1}^{-} \tilde{\nu}_{e}\right)$ for $m_{0}=50 \mathrm{GeV}$. The thick lines bound the regions of an invisible sneutrino similar as in Fig. 2 .

(b) Contours (0.025 pb, $0.05 \mathrm{pb}, 0.1 \mathrm{pb}, 0.2 \mathrm{pb}, 0.3 \mathrm{pb}$ and $0.4 \mathrm{pb})$ of $\sigma\left(e^{-} \gamma \rightarrow \tilde{\chi}_{2}^{-} \tilde{\nu}_{e}\right)$ for $m_{0}=50 \mathrm{GeV}$.

(c) Contours (0.1 pb, $0.25 \mathrm{pb}, 0.5 \mathrm{pb}, 0.75 \mathrm{pb}, 1.0 \mathrm{pb}$ and $1.25 \mathrm{pb})$ of $\sigma\left(e^{-} \gamma \rightarrow \tilde{\chi}_{1}^{-} \tilde{\nu}_{e}\right)$ for $m_{0}=200 \mathrm{GeV}$.

(d) Contours (0.025 pb, $0.05 \mathrm{pb}, 0.1 \mathrm{pb}, 0.2 \mathrm{pb}$ and $0.3 \mathrm{pb})$ of $\sigma\left(e^{-} \gamma \rightarrow \tilde{\chi}_{2}^{-} \tilde{\nu}_{e}\right)$ for $m_{0}=200 \mathrm{GeV}$. 


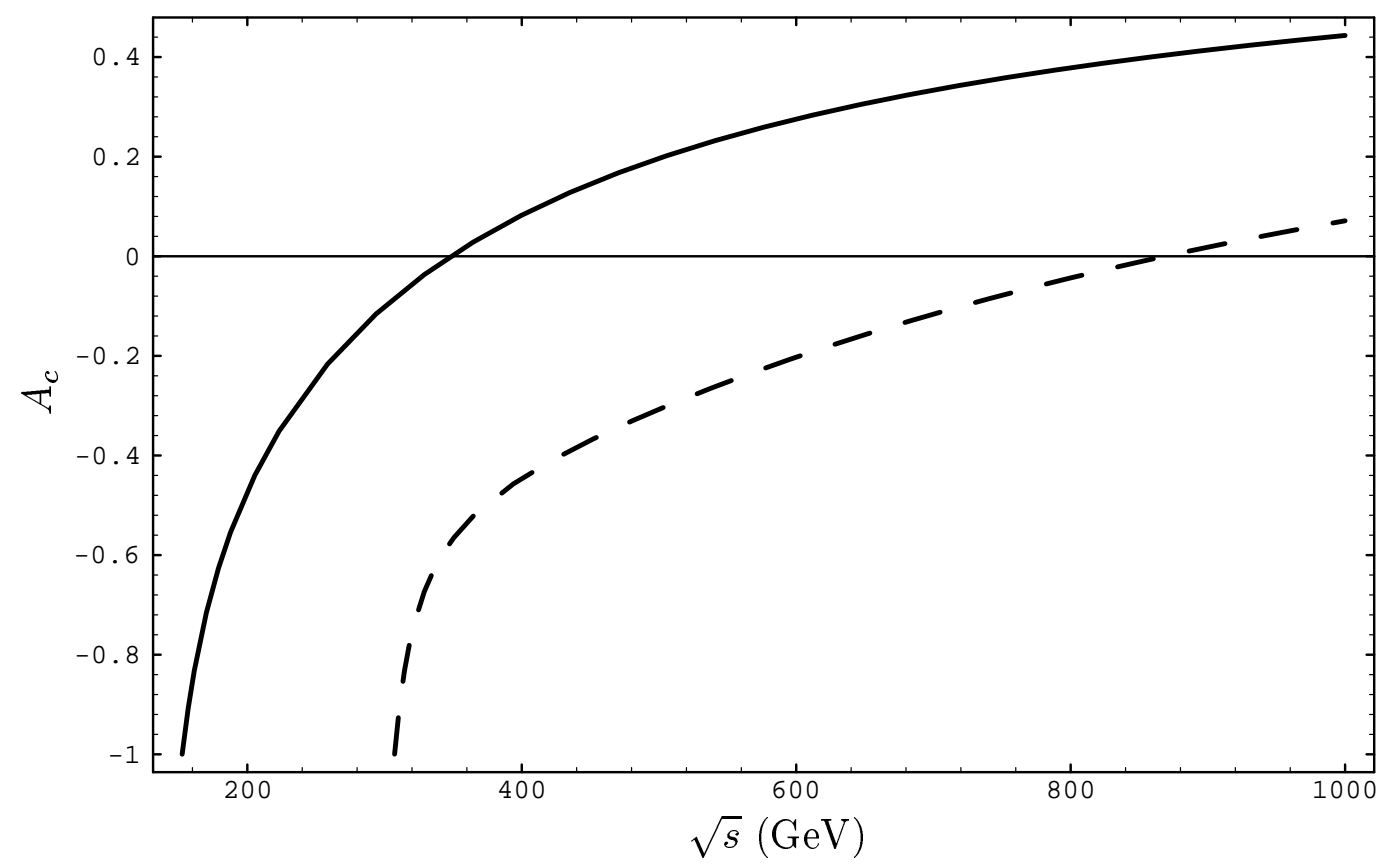

FIG. 7. Asymmetry $A_{c}$ in scenario A with $m_{0}=50 \mathrm{GeV}$ for production of $\tilde{\chi}_{1}^{-}(-)$and $\tilde{\chi}_{2}^{-}(--)$.

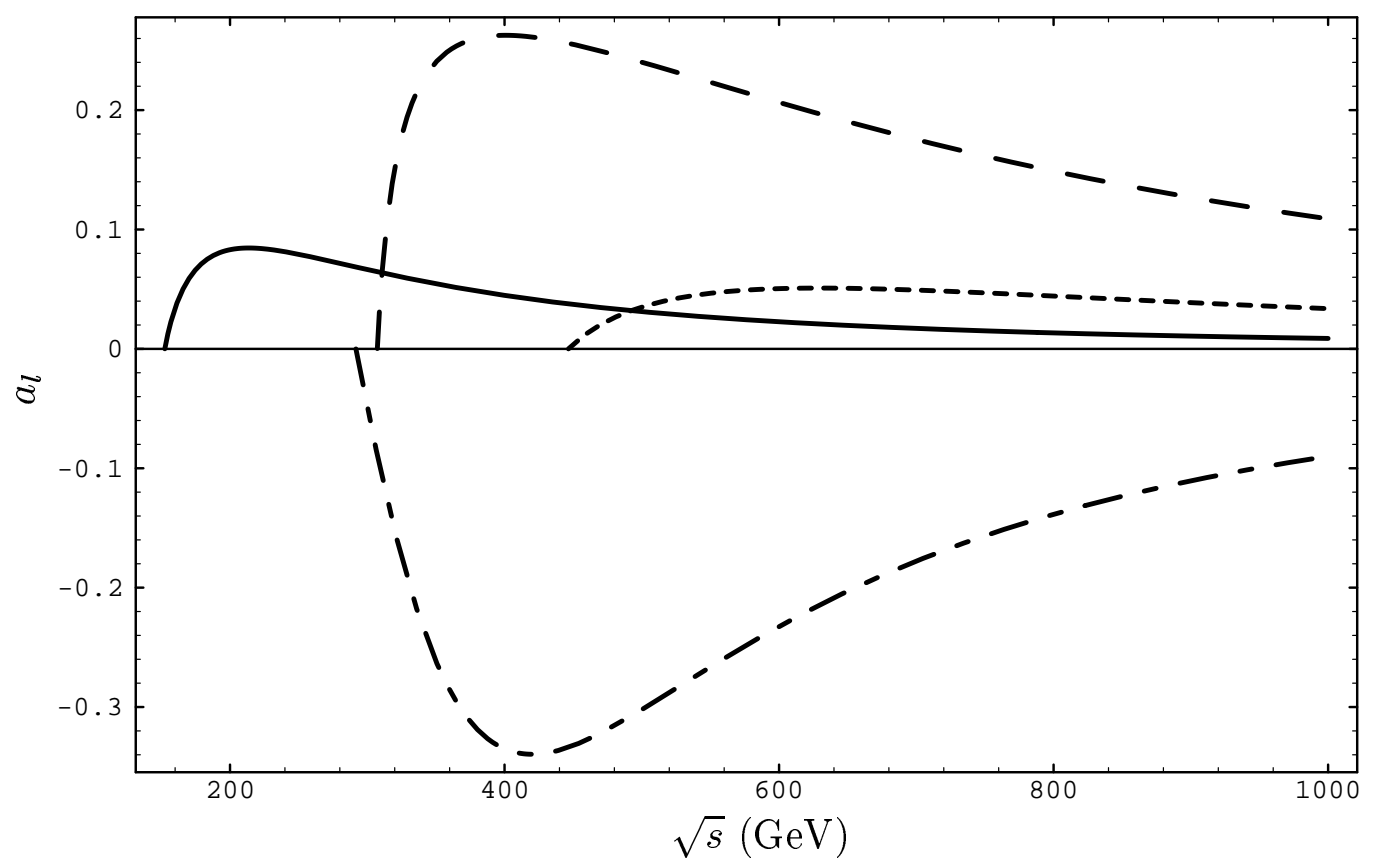

FIG. 8. Asymmetry $a_{l}$ in scenario A with $\cos \theta=0.5\left(\theta=60^{\circ}\right)$ for production of $\tilde{\chi}_{1}^{-}(-\square)$ and $\tilde{\chi}_{2}^{-}(--)$with $m_{0}=50 \mathrm{GeV}$ and for production of $\tilde{\chi}_{1}^{-}(---)$and $\tilde{\chi}_{2}^{-}\left(---_{-}\right)$with $m_{0}=200 \mathrm{GeV}$. 

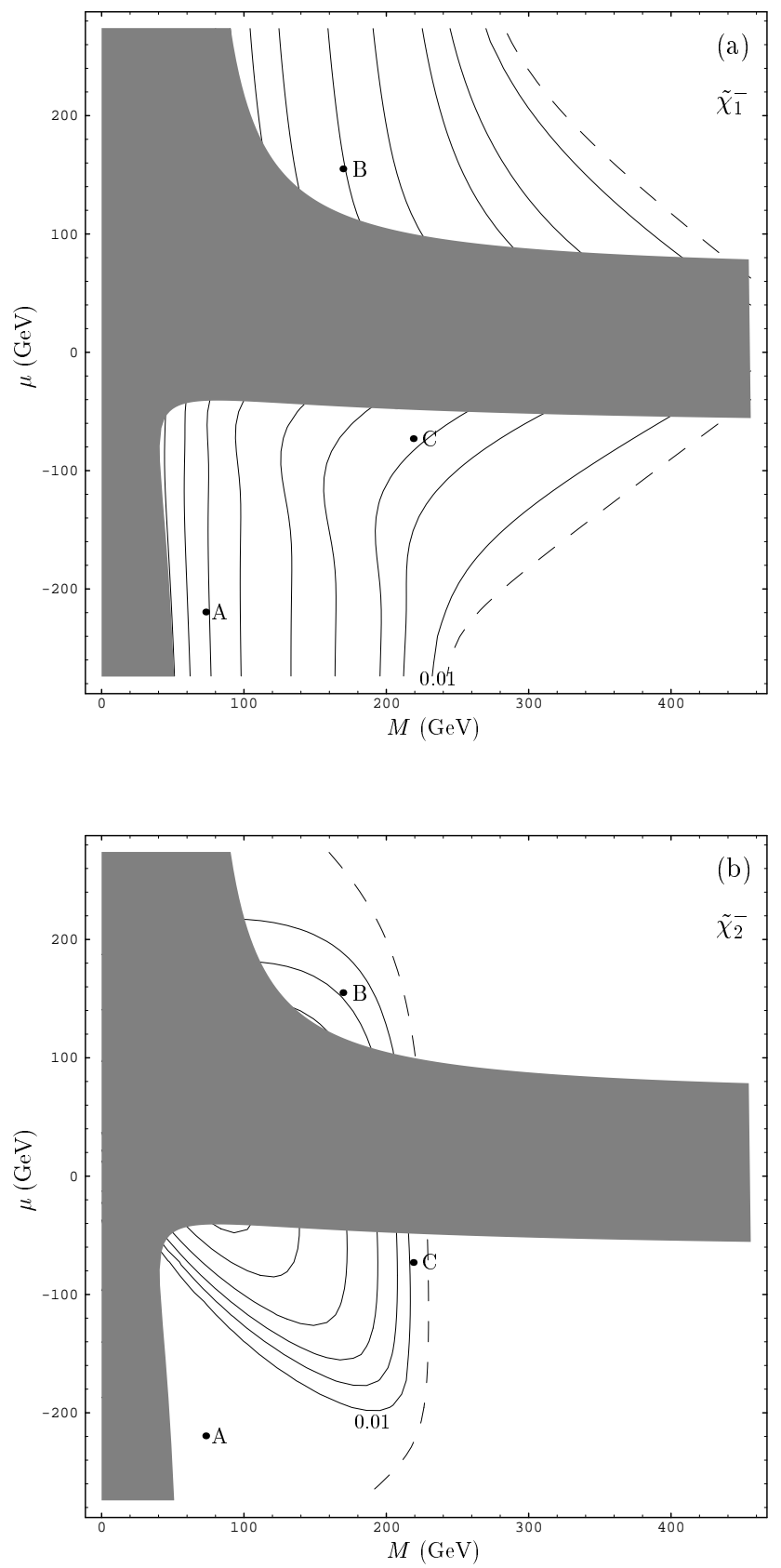

FIG. 9. Contours of the total cross section for $m_{0}=50 \mathrm{GeV}, x=4.83, \sqrt{s_{e e}}=500 \mathrm{GeV}$ and $\tan \beta=2$. The dashed lines mark the boundary $m_{\tilde{\chi}_{1,2}^{ \pm}}+m_{\tilde{\nu}_{e}}=414 \mathrm{GeV}$. The experimentally excluded domain is shaded.

(a) Contours (0.01 pb, $0.05 \mathrm{pb}, 0.1 \mathrm{pb}, 0.25 \mathrm{pb}, 0.5 \mathrm{pb}, 1.0 \mathrm{pb}, 1.5 \mathrm{pb}, 2.0 \mathrm{pb}$ and $2.5 \mathrm{pb})$ of $\sigma\left(e e \rightarrow \tilde{\chi}_{1}^{-} \tilde{\nu}_{e}\right)$.

(b) Contours (0.01 pb, $0.025 \mathrm{pb}, 0.05 \mathrm{pb}, 0.1 \mathrm{pb}, 0.2 \mathrm{pb}$ and $0.3 \mathrm{pb})$ of $\sigma\left(e e \rightarrow \tilde{\chi}_{2}^{-} \tilde{\nu}_{e}\right)$. 


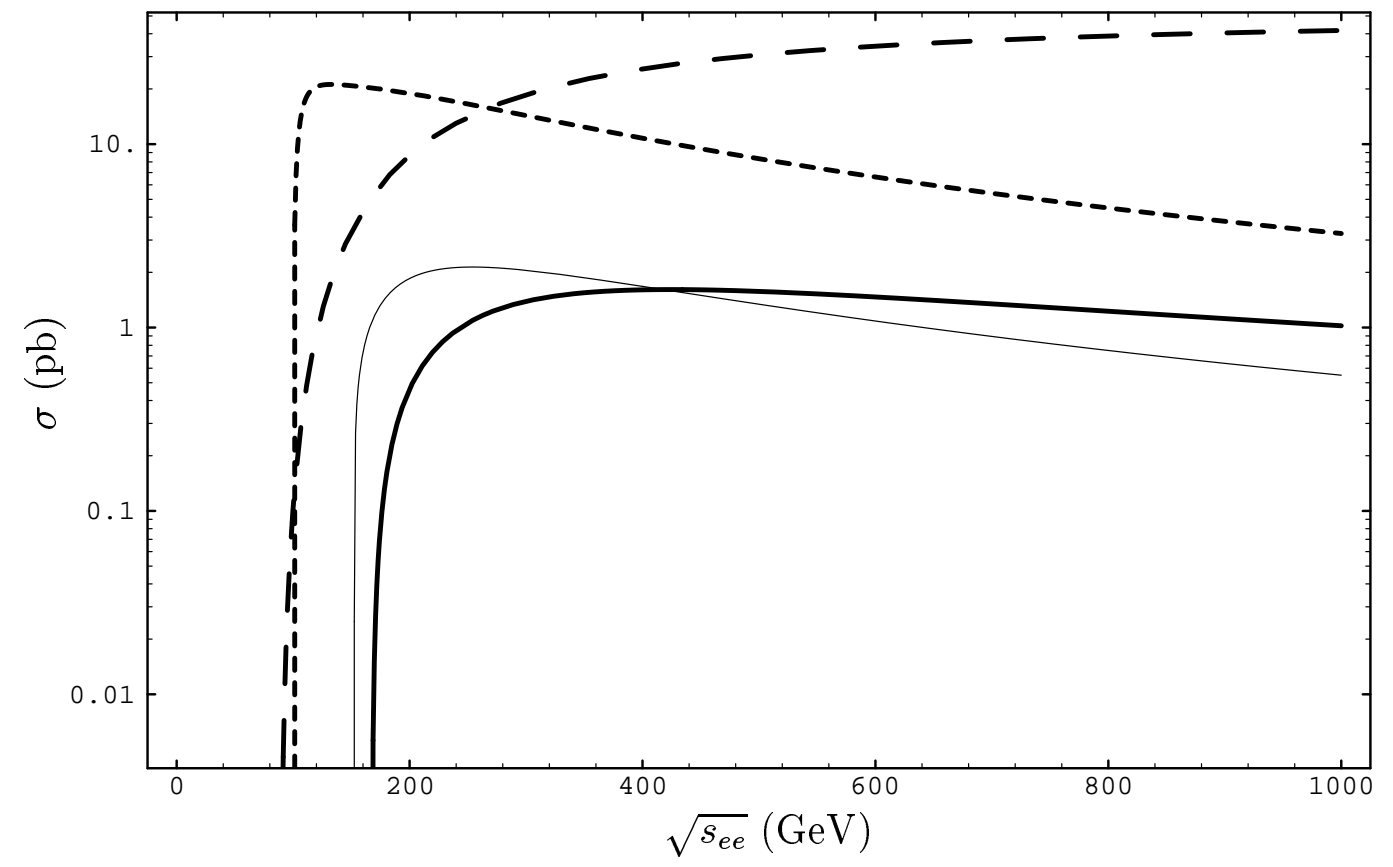

FIG. 10. Total cross sections $\sigma\left(s_{e e}\right)$ for $e e \rightarrow \tilde{\chi}_{1}^{-} \tilde{\nu}_{e}(-)$, ee $\rightarrow W \nu_{e}(--)$ and $e e \rightarrow e Z$ $(----)$ in scenario A with $m_{0}=50 \mathrm{GeV}, x=4.83$ and unpolarized electron and laser beams. For comparison we also show $\sigma\left(s_{e \gamma}\right)$ for $e \gamma \rightarrow \tilde{\chi}_{1}^{-} \tilde{\nu}_{e}$ with unpolarized beams (thin line). 

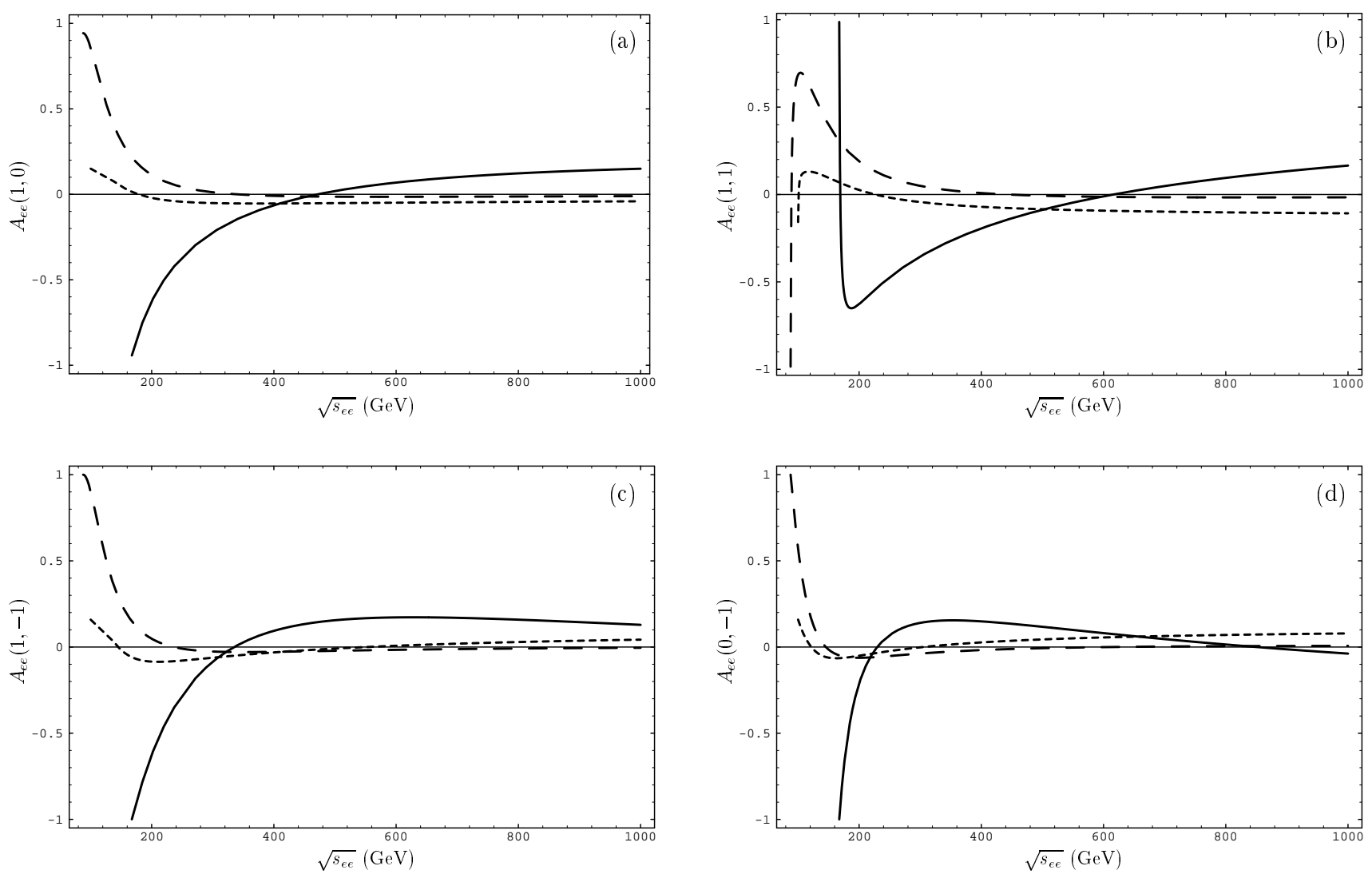

FIG. 11. Asymmetries of the convoluted cross sections for $e e \rightarrow \tilde{\chi}_{1}^{-} \tilde{\nu}_{e}(-)$, ee $\rightarrow W \nu_{e}$ $(-\quad-)$ and $e e \rightarrow e Z(----)$ in scenario A with $m_{0}=50 \mathrm{GeV}$ and $x=4.83$. 


\section{TABLES}

TABLE I. Chargino masses $m_{\tilde{\chi}_{j}^{ \pm}}(j=1,2)$ and mixing parameters $V_{i j}$ in three different scenarios of the parameters $M$ and $\mu$. Also shown are the masses of the sneutrinos for the two values of $m_{0}$ in each scenario, the masses of the lightest and the second lightest neutralino and the state of the lightest neutralino $\tilde{\chi}_{1}^{0}$ in terms of the weak eigenstates $\left(\tilde{\gamma}, \tilde{Z}, \tilde{H}_{a}^{0}, \tilde{H}_{b}^{0}\right)$. The $\eta_{\tilde{\chi}_{j}^{ \pm}}$ and $\eta_{\tilde{\chi}_{j}^{0}}$ denote the sign of the corresponding eigenvalues of the mass matrices of charginos and neutralinos, respectively.

\begin{tabular}{|c|c|c|c|}
\hline Scenario & $\mathrm{A}$ & $\mathrm{B}$ & $\mathrm{C}$ \\
\hline$\overline{M(\mathrm{GeV})}$ & +73.16 & +169.52 & +218.93 \\
\hline$\mu(\mathrm{GeV})$ & -219.47 & +155.04 & -72.96 \\
\hline$m_{0}(\mathrm{GeV})$ & 200 & 200 & 200 \\
\hline$\overline{m_{\tilde{\nu}_{e}}(\mathrm{GeV})}$ & 204.3 & 245.4 & 274.5 \\
\hline$\overline{m_{\tilde{\chi}_{1}^{ \pm}}, \eta_{\tilde{\chi}_{1}^{ \pm}}}$ & $87.4 \mathrm{GeV},+1$ & $88.0 \mathrm{GeV},-1$ & $87.3 \mathrm{GeV},+1$ \\
\hline$m_{\tilde{\chi}_{2}^{ \pm}}, \eta_{\tilde{\chi}_{2}^{ \pm}}$ & $242.3 \mathrm{GeV},+1$ & $240.5 \mathrm{GeV},+1$ & $241.8 \mathrm{GeV},+1$ \\
\hline$V_{11}=V_{22}$ & +0.9974 & +0.7279 & +0.3956 \\
\hline$V_{21}=-V_{12}$ & -0.0727 & +0.6857 & +0.9184 \\
\hline$\overline{m_{\tilde{\chi}_{1}^{0}}, \eta_{\tilde{\chi}_{1}^{0}}}$ & $40.0 \mathrm{GeV},+1$ & $54.1 \mathrm{GeV},+1$ & $67.3 \mathrm{GeV},+1$ \\
\hline$m_{\tilde{\chi}_{2}^{0}}, \eta_{\tilde{\chi}_{2}^{0}}$ & $88.2 \mathrm{GeV},+1$ & $113.2 \mathrm{GeV},+1$ & $99.7 \mathrm{GeV},-1$ \\
\hline State of the $\tilde{\chi}_{1}^{0}$ & $(-0.95,0.30,0.08,0.08)$ & $(-0.48,0.66,-0.51,-0.27)$ & $(-0.13,0.19,-0.20,0.95)$ \\
\hline
\end{tabular}

